\title{
Navigating surgical fluorescence cameras using near-infrared optical tracking
}

Matthias N. van Oosterom

David A. den Houting

Cornelis J. H. van de Velde

Fijs W. B. van Leeuwen 


\title{
Navigating surgical fluorescence cameras using near-infrared optical tracking
}

\author{
Matthias N. van Oosterom, ${ }^{a, b}$ David A. den Houting, ${ }^{a}$ Cornelis J. H. van de Velde, ${ }^{b}$ and \\ Fijs W. B. van Leeuwen ${ }^{a, c, *}$ \\ aLeiden University Medical Center, Interventional Molecular Imaging Laboratory, Department of Radiology, Leiden, The Netherlands \\ 'Leiden University Medical Center, Department of Surgery, Leiden, The Netherlands \\ 'The Netherlands Cancer Institute-Antoni van Leeuwenhoek Hospital, Department of Urology, Amsterdam, The Netherlands
}

\begin{abstract}
Fluorescence guidance facilitates real-time intraoperative visualization of the tissue of interest. However, due to attenuation, the application of fluorescence guidance is restricted to superficial lesions. To overcome this shortcoming, we have previously applied three-dimensional surgical navigation to position the fluorescence camera in reach of the superficial fluorescent signal. Unfortunately, in open surgery, the near-infrared (NIR) optical tracking system (OTS) used for navigation also induced an interference during NIR fluorescence imaging. In an attempt to support future implementation of navigated fluorescence cameras, different aspects of this interference were characterized and solutions were sought after. Two commercial fluorescence cameras for open surgery were studied in (surgical) phantom and human tissue setups using two different NIR OTSs and one OTS simulating light-emitting diode setup. Following the outcome of these measurements, OTS settings were optimized. Measurements indicated the OTS interference was caused by: (1) spectral overlap between the OTS light and camera, (2) OTS light intensity, (3) OTS duty cycle, (4) OTS frequency, (5) fluorescence camera frequency, and (6) fluorescence camera sensitivity. By optimizing points 2 to 4 , navigation of fluorescence cameras during open surgery could be facilitated. Optimization of the OTS and camera compatibility can be used to support navigated fluorescence guidance concepts. ๑ 2018 Society of Photo-Optical Instrumentation Engineers (SPIE) [DOI: 10.1117/1. JBO.23.5.056003]
\end{abstract}

Keywords: surgical navigation; fluorescence-guided surgery; augmented reality; optical tracking; computer-assisted surgery.

Paper 170768R received Dec. 7, 2017; accepted for publication Apr. 9, 2018; published online May 9, 2018.

\section{Introduction}

Fluorescence guidance has demonstrated value in the field of oncologic surgery where it helps provide real-time visualization of the lesion of interest. ${ }^{1}$ Due to its depth restrictions, however, the utility of fluorescence guidance is currently limited to superficial lesions. ${ }^{2}$ To compensate for this shortcoming, we have previously introduced the clinical use of dual-modality or rather hybrid tracers. ${ }^{3}$ These tracers directly link preoperative identification of radioactive lesions at the department of nuclear medicine [e.g., using three-dimensional (3-D) single-photon emission computed tomography/x-ray computed tomography (SPECT/CT) or positron emission tomography/CT (PET/CT) data sets] to the intraoperative detection of fluorescent lesions. Through this connection, it has become possible to create surgical navigation setups that realize positional tracking of fluorescence cameras in pre- or intraoperative 3-D imaging data sets. ${ }^{4-6}$ Combined with futuristic virtual and augmented reality-based computer-assisted surgery techniques, these approaches extend the use of fluorescence guidance toward lesions that lie beyond the depth-related fluorescence detection limits (i.e., $>1 \mathrm{~cm}$ deep).

Initial studies, using a near-infrared (NIR) optical tracking system (OTS) and tracking fiducials placed on both the fluorescence camera and the patient, helped to demonstrate the potential of this navigated fluorescence camera concept in a variety of settings. ${ }^{4,5,7,8}$ Unfortunately, in some of the open surgical

*Address all correspondence to: Fijs W. B. van Leeuwen, E-mail: f.w.b. van_leeuwen@lumc.nl procedures, the OTS caused an interfering flickering signal that prevented fluorescence imaging (see Figs. 1-3). ${ }^{4}$ Intriguingly, we observed that this OTS interference differed substantially between the two open surgery cameras we have studied; $;$, while the interference completely obstructed fluorescence imaging with one system, the effect was only minor for navigation of the other system. Therefore, the aim of this study was to investigate, which features influenced the interference and to identify possible engineering solutions to support further implementation of the navigation concept with fluorescence cameras.

\section{Methods}

\subsection{Navigation and Tracking Systems}

Our "standard" navigation setup was based on the declipse SPECT navigation device (SurgicEye GmbH, Munich, Germany; see Fig. 1). This navigation system incorporates a Polaris Vicra OTS (OTS ${ }^{\text {vicra, }}$ Northern Digital Inc., Waterloo, Canada) to allow for object position and orientation tracking of geometrically unique reference targets using a fixed pulsed NIR light source [20 Hz; center wavelength (CWL) of $863 \mathrm{~nm}$; full-width-at-halfmaximum (FWHM) of $49 \mathrm{~nm}] .{ }^{4,9}$ The pulse on-time per period was about $1.8 \mathrm{~ms}$, resulting in a duty cycle of roughly $4 \%$ (see Appendix A).

Next to the declipseSPECT integrated OTS ${ }^{\text {vicra }}$, a prototype OTS (OTS ${ }^{\text {orthos }}$; Orthos prototype, Advanced Real-time Tracking $\mathrm{GmbH}$, Weilheim i.OB, Germany) was used. This system

$1083-3668 / 2018 / \$ 25.00$ @ 2018 SPIE 

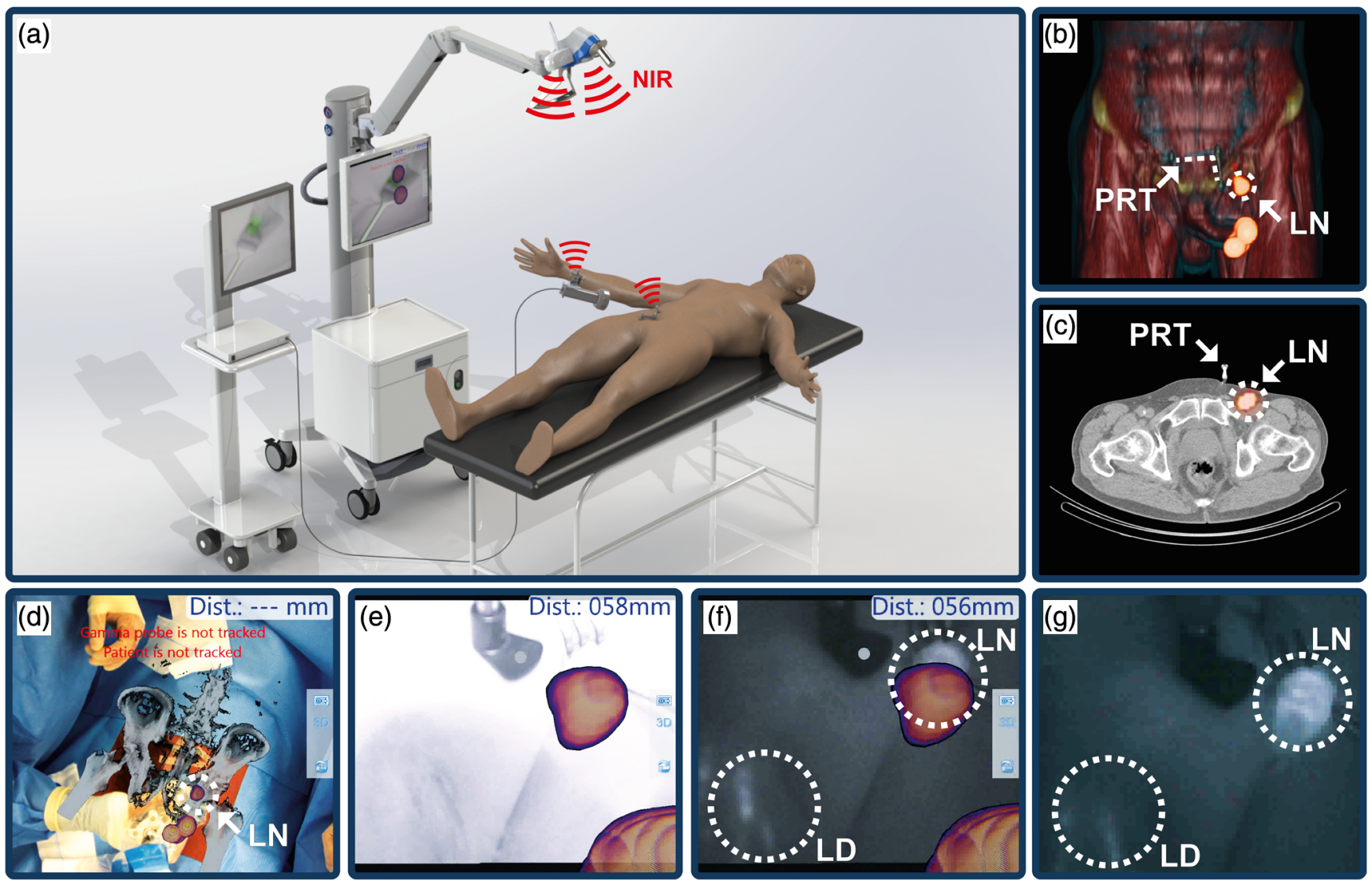

Fig. 1 Illustration of in vivo navigated fluorescence camera during penile cancer-related sentinel lymph node biopsy. (a) Overview of navigated setup in the operating room. (b) 3-D rendering of SPECT/CT patient scan, with patient tracking fiducials (PRT) and lymph node targeted for navigation (LN) visible. (c) two-dimensional slice showing the navigated LN. (d) Augmented reality overlay of the patient scan in the operating room. (e) Augmented reality overlay of the patient scan in the fluorescence camera video feed, displaying the LN location (purple) and the estimated distance toward it. Due to the OTS interference, fluorescence imaging is obstructed, predominantly showing white background in the video feed. (f) Same navigated fluorescence camera image without interference, displaying the LN and lymph ducts (LD). (g) Fluorescence imaging only, without interference.

allows for manual customization of the pulse frequencies in the 10 to $60 \mathrm{~Hz}$ range (CWL: $885 \mathrm{~nm}$, FWHM: $108 \mathrm{~nm}$ ). The pulseon time per period was about $0.4 \mathrm{~ms}$, which results in a duty cycle of $0.8 \%$ at $20 \mathrm{~Hz}$. To allow for automatic triggering of

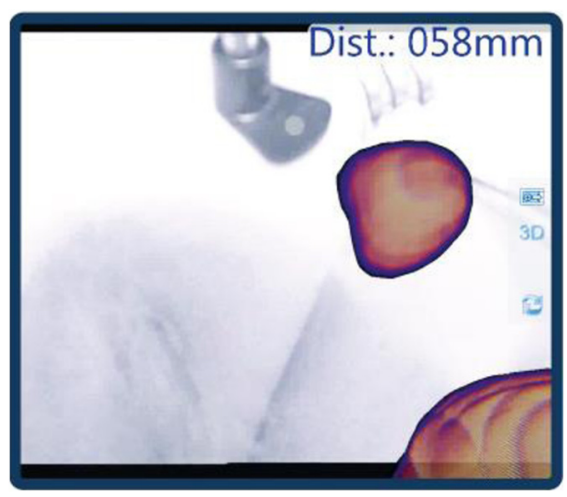

Fig. 2 Video example of OTS-induced interference during in vivo navigation of fluorescence camera for penile cancer-related sentinel lymph node biopsy. With this fluorescence camera, interference completely obstructs fluorescence imaging. Fluorescence and interference signals are both in white (Video 1, MPEG, 7.5 MB [URL: https://doi.org/10.1117/1.JBO.23.5.056003.1]). the pulse frequency, either an analog video signal or digital transistor-transistor-logic signal could be connected via the Bayonet Neill-Concelman (BNC) input connector.

In addition to both these OTSs, a light-emitting diode (LED)based illumination setup was created, simulating the light source of an OTS. This allowed for controlled variations to be made in both the tracking pulse frequency and duty cycle during the experiments. This configuration consisted of three $850 \mathrm{~nm}$ LEDs (CWL: 845 nm, FWHM: 33 nm; HE1-220AC, Harvatek Corp., Hsinchu City, Taiwan) connected to a multichannel universal LED controller (Mightex Systems, Pleasanton, California).

\subsection{Fluorescence Camera Systems}

Two open surgery conformité européenne (CE) marked fluorescence cameras were used for this study. First, a PDE camera (Hamamatsu Photonics K.K., Hamamatsu, Japan; European specifications) using a ring of LEDs (CWL: $755 \mathrm{~nm}$, FWHM: $10 \mathrm{~nm}$ ) to excite the NIR fluorescent dye indocyanine green (ICG). The light detected by this charge-coupled device (CCD) camera is filtered by a $>820 \mathrm{~nm}$ long pass filter. ${ }^{10}$ The resulting video-feed was delivered as an analog $50 \mathrm{~Hz}$ interlaced video signal. Second, a VITOM exoscopic camera setup (IMAGE1 S H3-Z FI Three-Chip FULL HD camera head and VITOM ${ }^{\circledR}$ II 


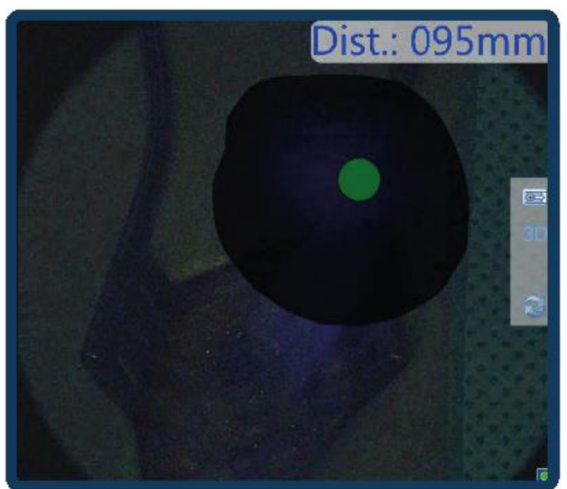

Fig. 3 Video example of OTS-induced interference during in vivo navigation of fluorescence camera for penile cancer-related sentinel lymph node biopsy. With this fluorescence camera, interference is clearly visible when low fluorescence intensities are observed. However, fluorescence imaging is still possible. Fluorescence and interference signals are both in blue (Video 2, MPEG, 7.8 MB [URL: https://doi.org/10.1117/1.JBO.23.5.056003.2]).

NIR/ICG Telescope 0 deg; KARL STORZ Endoskope GmbH \& Co. KG, Tuttlingen, Germany; European specifications). In this setup, fluorescence excitation occurs using a prototype D-LIGHT P filtered Xenon light source (CWL: $\sim 740 \mathrm{~nm}$, FWHM: $120 \mathrm{~nm}) .{ }^{11}$ The light collected by the CCD camera was filtered to allow light in the 390 to $670 \mathrm{~nm}$ and $>800 \mathrm{~nm}$ range to be collected. ${ }^{12}$ The resulting video-feed was delivered as a digital $50 \mathrm{~Hz}$ progressive video signal. A summary of the fluorescence camera and tracking system characteristics is shown in Table 1 and Fig. 4.

\subsection{Surgical Fluorescence Phantom and Human Tissue}

A surgical fluorescence phantom was fabricated for the experiments. This setup consisted of a previously described, siliconebased phantom ${ }^{8}$ with two fluorescent beads incorporated (see Appendix B). In addition, surgically excised human tissue was used. ICG-nanocolloid (dissolved in saline) was prepared as previously described. ${ }^{13}$ Four $0.1 \mathrm{~mL}$ injections, with effectively $40 \mu \mathrm{g} / \mathrm{mL}$ ICG, where used for the injection site, where $0.1 \mathrm{~mL}$ with $1 \%$ of the injected dose was used for the sentinel lymph node. ${ }^{13}$

\subsection{Characterization of the Tracking Interference}

All fluorescence camera recordings were performed using an Epiphan frame grabber (DVI2PCIe, Epiphan Systems Inc., Ottawa, Ontario, Canada) integrated within the declipseSPECT navigation system. To quantify the OTS-induced interference, the acquired recordings were analyzed using MATLAB $^{\circledR}$ (The MathWorks Inc., Natick, Massachusetts). To evaluate the intensity of the interference signals in the fluorescence imaging recordings, the mean pixel intensity (MPI) per video frame was calculated

$\operatorname{MPI}(t)=\frac{1}{N} \sum_{i=1}^{N} p_{i}(t)$.

With MPI in counts, $t$ is the video frame number, $p_{i}$ is the individual pixel value, and $N$ is the total number of pixels in one frame. To quantify the dynamic character of these interference patterns, a "severity of dynamic interference value" (SDIV) was proposed. To include rapid changes in intensity, while excluding gradual changes, this SDIV was formulated as the standard deviation $(\sigma)$ of the first derivative of MPI; i.e., a stable video illumination would result in a low SDIV while a highly dynamic flickering in the video would result in a high SDIV

$\mathrm{SDIV}=\sigma_{\mathrm{MPI}^{\prime}}=\sqrt{\frac{1}{J} \sum_{t=1}^{J}\left[\mathrm{MPI}^{\prime}(t)-\overline{\left.\mathrm{MPI}^{\prime}\right]^{2}} .\right.}$

With SDIV in counts/frame or counts/s, $J$ is the total number of video frames, MPI' is the first derivative of MPI, and $\overline{\mathrm{MPI}^{\prime}}$ is the average thereof.

Table 1 Characteristics of the fluorescent dye and devices used.

\begin{tabular}{|c|c|c|c|c|c|}
\hline Light source & Emission spectrum & Frequency & Pulse-on-time & & \\
\hline ICG (in albumin) & $\begin{array}{l}\text { CWL: } 818 \mathrm{~nm} \\
\text { (FWHM: } 40 \mathrm{~nm} \text { ) }\end{array}$ & Continuous $^{a}$ & $-^{a}$ & & \\
\hline OTS vicra & $\begin{array}{l}\text { CWL: } 863 \mathrm{~nm} \\
\text { (FWHM: } 49 \mathrm{~nm} \text { ) }\end{array}$ & $20 \mathrm{~Hz}$ & $\sim 1.8 \mathrm{~ms}$ & & \\
\hline OTS orthos & $\begin{array}{l}\text { CWL: } 885 \mathrm{~nm} \\
\text { (FWHM: } 108 \mathrm{~nm} \text { ) }\end{array}$ & $\begin{array}{c}\text { Modular } \\
(10 \text { to } 60 \mathrm{~Hz})\end{array}$ & $\sim 0.4 \mathrm{~ms}$ & & \\
\hline LED setup & $\begin{array}{l}\text { CWL: } 845 \mathrm{~nm} \\
\text { (FWHM: } 33 \mathrm{~nm} \text { ) }\end{array}$ & Modular & Modular & & \\
\hline Camera & $\begin{array}{l}\text { Excitation light } \\
\text { source }\end{array}$ & $\begin{array}{l}\text { ICG emission } \\
\text { filter }\end{array}$ & $\begin{array}{c}\text { Camera } \\
\text { frame rate }\end{array}$ & $\begin{array}{l}\text { Measured camera } \\
\text { recording frequency }\end{array}$ & $\begin{array}{l}\text { Sensor } \\
\text { type }\end{array}$ \\
\hline PDE (EU version) & $\begin{array}{l}\text { LED-CWL: } 755 \mathrm{~nm} \\
\text { (FWHM: } 10 \mathrm{~nm} \text { ) }\end{array}$ & $>820 \mathrm{~nm}$ & $50 \mathrm{~Hz}$ interlaced & $25 \mathrm{~Hz}$ & CCD-based \\
\hline VITOM (EU version) & $\begin{array}{l}\text { Xenon light bulb } \\
\text { (D-light P version)-CWL: } \\
740 \mathrm{~nm} \text { (FWHM: } 120 \mathrm{~nm} \text { ) }\end{array}$ & $\begin{array}{l}<670 \mathrm{~nm} \text { and } \\
\sim>820 \mathrm{~nm}\end{array}$ & $50 \mathrm{~Hz}$ progressive & $25 \mathrm{~Hz}$ & CCD-based \\
\hline
\end{tabular}

as depended on the excitation source used, which is mainly continuous. 


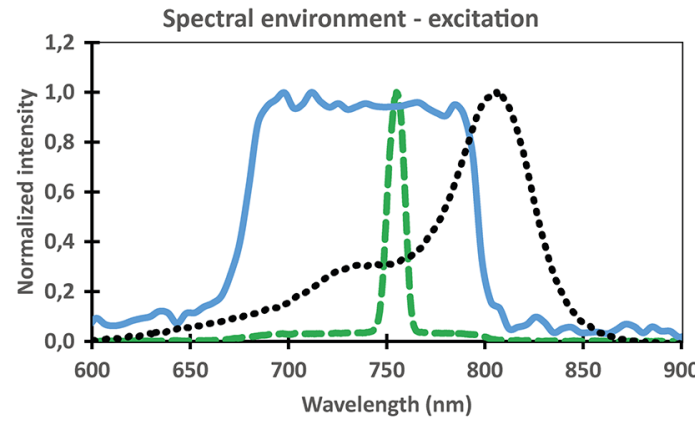

(a)

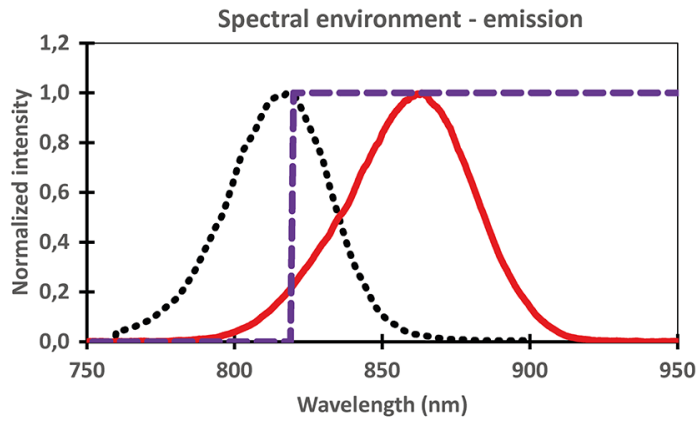

$\ldots$... ICG emission —OTS - -PDE and VITOM transmission

(b)

Fig. 4 Spectral environment. (a) The excitation light used by the PDE and VITOM camera and the ICG absorption spectrum. (b) The ICG spectrum and the light spectrum employed by the OTS vicra. Spectral transmission window of the PDE and VITOM is estimated from the literature. ${ }^{10,12}$

\subsection{Interference Effect for a Modular Tracking Frequency and Duty Cycle}

To quantitatively measure the effect that a variable OTS pulse frequency and duty cycle have on the observed interference during fluorescence imaging, the LEDs were used. To generate light reflection, the LED setup was pointed at a flat surface using a $15 \mathrm{~cm}$ distance and the camera systems were placed at a $17 \mathrm{~cm}$ distance (Fig. 5). These distances were chosen to maximize the captive nature of the most sensitive setup while preventing saturation of the camera CCD chips. At each LED setting (frequencies: 5 to $100 \mathrm{~Hz}$, duty cycles: $1 \%$ to $90 \%$ ), 2 s video recordings were generated and analyzed (i.e., MPI and SDIV values) for both fluorescence cameras. These experiments were performed in duplicate.

\subsection{Interference in a Surgical Phantom and Human Tissue}

To simulate surgical application, the two navigated fluorescence camera systems were sequentially positioned right above the surgical phantom using a distance of $15 \mathrm{~cm}$. Subsequently, either the OTS ${ }^{\text {vicra }}$ or OTS ${ }^{\text {orthos }}$ was also placed above the surgical phantom using a distance of 130 or $30 \mathrm{~cm}$. To illustrate the difference in interference in the "standard" navigation setup, video recordings were produced and analyzed (i.e., MPI and
SDIV values) with the OTS ${ }^{\text {vicra }}$ turned off and on. Furthermore, using the BNC synchronization input of the OTS ${ }^{\text {orthos, }}$ the video signal of the PDE was sampled, allowing for synchronization of the tracking frequency with the PDE acquisition frequency. To illustrate the effect of synchronized NIR optical tracking during surgery, video recordings were acquired with the OTS $^{\text {orthos }}$ turned off and on, using a tracking frequency of 20 (unsynchronized) and $25 \mathrm{~Hz}$ (synchronized). These recordings were also analyzed for the above-mentioned MPI and SDIV values. Furthermore, the most important measurements, i.e., PDE fluorescence imaging with "no tracking," "OTS ${ }^{\text {vicra }}$ track-

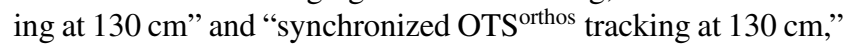
were also recorded using the human tissue.

\subsection{Gated Tracking Pulse and Fluorescence Imaging for PDE}

To create an alternating gated setup, a tracking pulse should only be sent whenever there is no fluorescence image capture (Fig. 6). We assumed the PDE camera would have a "dead-imagingmoment" long enough to send out this tracking pulse, without it being detected by the camera. This was investigated by building and programming a small electronic circuit to sample the frequency timing of the PDE video signal and trigger the OTS $^{\text {orthos }}$ tracking pulse. This circuit was built around an
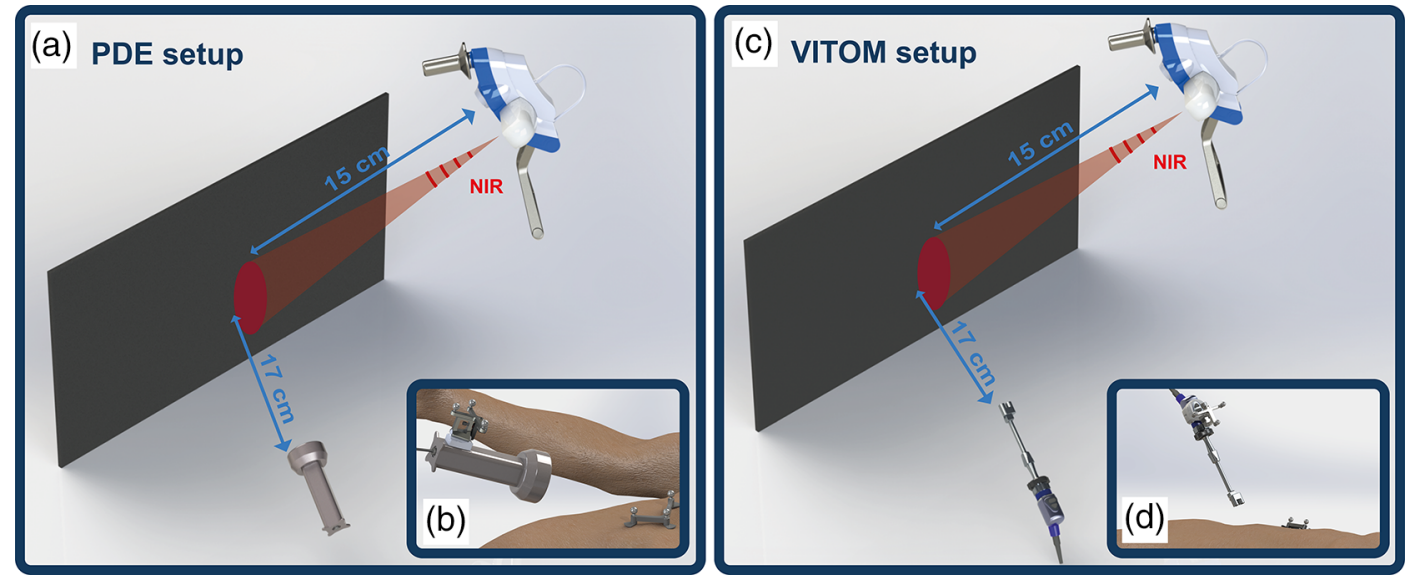

Fig. 5 Schematic overview of the measurements with the LED setup. (a) Setup for the PDE camera and LED setup, both pointed at a black surface. (b) Analogy of the clinical usage of the navigated PDE. (c) Setup for the VITOM and LED setup, both pointed at the black surface. (d) Analogy of the clinical usage of the navigated VITOM. 


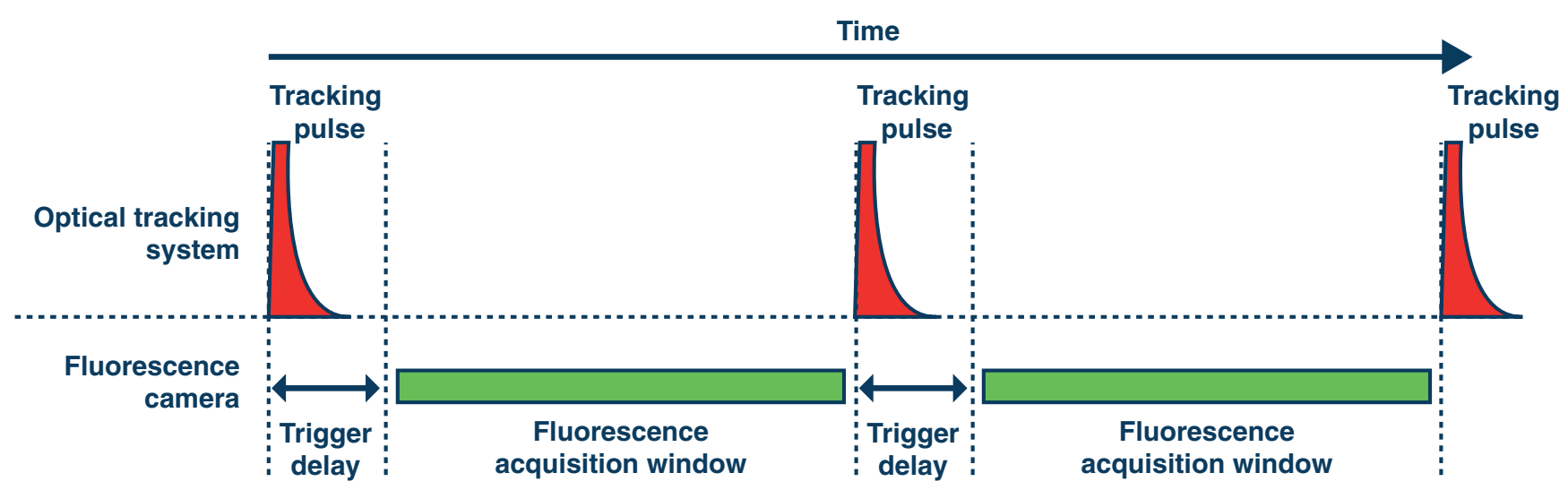

Fig. 6 Gated tracking pulse and fluorescence imaging. In a sequential fashion, optical tracking pulses are only emitted when the fluorescence camera is not acquiring a fluorescence image.

LM1881N video processing chip and an Arduino Uno microcontroller (Arduino AG, Italy).

\section{Results}

\subsection{Interference in a Surgical Phantom Setup}

Table 2 and Appendix C demonstrate the interference the $20 \mathrm{~Hz}$ OTS $^{\text {vicra }}$ induces for the different fluorescence cameras. This interference is the result of the light emitted from the OTS, overlapping with the spectral detection window for which NIR fluorescence cameras are traditionally designed (>800 nm). More specifically, the spectral emission of the OTS overlaps with the fluorescence emission of the clinically approved NIR dye ICG (emission max at $820 \mathrm{~nm}$ ) and more experimental Cy7-analogs (emission max between 795 and $825 \mathrm{~nm}$ ) (Fig. 4). ${ }^{4,14,15}$ For both systems, the MPI and SDIV values rose when the OTS ${ }^{\text {vicra }}$ was turned on (Table 2); a rise of 142.6 counts (PDE) versus 18.5 counts (VITOM) for the mean MPI values and 1757.5 counts/s (PDE) versus 410.0 counts/s (VITOM) for the SDIV values was recorded. As illustrated in Appendix C, the interference in the PDE setup was so severe that it inhibited the differentiation of the fluorescent beads with respect to their surroundings. Using the VITOM setup, the dynamic OTS ${ }^{\text {vicra }}$ light was also clearly visible; however, it did not limit the delineation of the fluorescent beads.

\subsection{Interference Effect for a Modular Tracking Frequency and Duty Cycle}

Next to the spectral overlap between the light emitted by the OTS and the spectral detection window of the surgical fluorescence cameras, exposure to the variations in LED light pulse frequencies and duty cycles influenced the interferences (Fig. 5). Plotting of the MPI and SDIV revealed the LED settings affected the two cameras differently (Fig. 7 and Appendix D). Of both cameras, the PDE proved most sensitive to the NIR LED light, yielding substantially higher MPI values at identical settings; e.g., a mean MPI of 59.3 counts for the PDE and 26.9 counts for the VITOM at a frequency of $20 \mathrm{~Hz}$ and duty cycle of $3.2 \%$. As can be seen in Appendix D, the intensity of the MPI decreased with decreasing duty cycles (i.e., duty cycles approaching 0\%). Figure 7 reveals the duty cycle settings also influenced the SDIV values, showing a decrease in the intensity of the dynamic interference when duty cycle values approached either $0 \%$ or $100 \%$. For both cameras, minimal dynamic interference (i.e., low SDIV value) was observed at tracking pulse frequencies of 25,50 , and $75 \mathrm{~Hz}$, which is in line with recording frame rates of 25 frames per second. Unfortunately, a stable interfering background illumination remained (i.e., stable nonzero MPI values). These results indicate that the mismatch between the frequency of the camera recording frame rate and the OTS tracking light pulse cause the dynamic character (flickering) of the interference.

\subsection{Synchronized Tracking Frequency for the PDE in Both Phantom and Human Tissue}

Given the above-reported findings, we reasoned that using an alternative OTS with controllable tracking frequency could help solve or reduce the severe flickering effects. Using the surgical phantom setup, Table 2 and Appendix C illustrate that a $20-\mathrm{Hz}$

Table 2 Quantitative analysis of the surgical fluorescence phantom measurements with the OTS ${ }^{\text {vicra }}$ and OTS ${ }^{\text {orthos }}$. Mean MPI and SDIV values calculated for the PDE and VITOM, with and without the OTS turned on at different settings and distances.

\begin{tabular}{|c|c|c|c|c|c|}
\hline Condition & $\begin{array}{c}\text { OTS } \\
\text { frequency } \\
(\mathrm{Hz})\end{array}$ & $\begin{array}{c}\text { OTS } \\
\text { distance } \\
(\mathrm{cm})\end{array}$ & $\begin{array}{c}\text { Mean } \\
\text { MPI } \\
\text { (counts) }\end{array}$ & $\begin{array}{l}\text { SDIV } \\
\text { (counts/ } \\
\text { frame) }\end{array}$ & $\begin{array}{c}\text { SDIV } \\
\text { (counts/s) }\end{array}$ \\
\hline \multicolumn{6}{|l|}{ OTS } \\
\hline $\begin{array}{l}\text { PDE without } \\
\text { OTS }\end{array}$ & - & - & 38.7 & 0.2 & 5.0 \\
\hline PDE with OTS & 20 & 130 & 181.3 & 70.5 & 1762.5 \\
\hline $\begin{array}{l}\text { VITOM without } \\
\text { OTS }\end{array}$ & - & - & 27.5 & 0.2 & 5.0 \\
\hline $\begin{array}{l}\text { VITOM with } \\
\text { OTS }\end{array}$ & 20 & 130 & 46.0 & 16.6 & 415.0 \\
\hline \multicolumn{6}{|l|}{ OTS ${ }^{\text {orthos }}$} \\
\hline $\begin{array}{l}\text { PDE without } \\
\text { OTS }\end{array}$ & - & - & 32.6 & 0.2 & 5.0 \\
\hline PDE with OTS & 20 & 130 & 42.2 & 2.6 & 65.0 \\
\hline PDE with OTS & 25 & 130 & 54.8 & 0.1 & 2.5 \\
\hline PDE with OTS & 20 & 30 & 88.0 & 40.7 & 1017.5 \\
\hline PDE with OTS & 25 & 30 & 147 & 0.3 & 7.5 \\
\hline
\end{tabular}



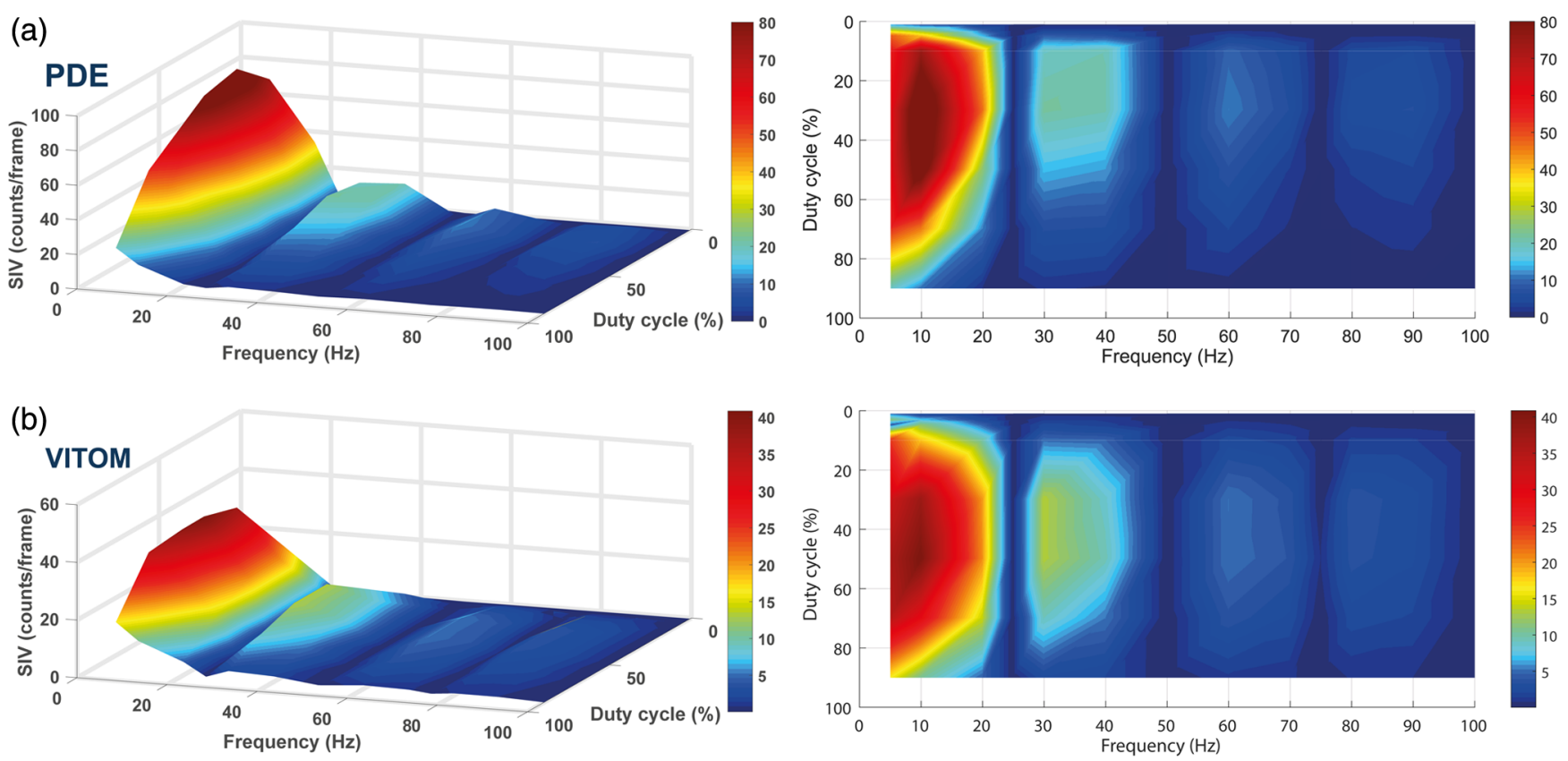

Fig. 7 Visualization of the severity of dynamic interference for the fluorescence cameras using the LED setup. (a) The SDIV results for the PDE in 3-D-view (left) or top-view (right). (b) The SDIV results for the VITOM in 3-D-view (left) or top-view (right).

OTS $^{\text {orthos }}$ already reduced the interference intensity fourfold compared to what was observed for the OTS ${ }^{\text {vicra }}$ : mean MPI values of 42.2 counts versus 181.3 counts, respectively. This improvement is most likely a combination of a shorter pulse-on time (i.e., a lower duty cycle) and a lower light intensity. When the OTS ${ }^{\text {orthos }}$ pulse frequency was matched with the PDE recording frequency (at $25 \mathrm{~Hz}$ ), SDIV values approached zero and the dynamic character of the interference was completely eliminated (Table 2 and Appendix C), thus providing comparable SDIV values with the OTS ${ }^{\text {orthos }}$ turned on and off. However, mean MPI values differed between 54.8 counts with the OTS ${ }^{\text {orthos }}$ turned on and 32.6 counts with the OTS ${ }^{\text {orthos }}$ turned off (distance of $130 \mathrm{~cm}$ ). Despite this stable background, differentiation between fluorescent beads and background in the phantom setup was possible. Figure 8 clearly shows that similar results are found when this concept is applied to human tissue; where fluorescence imaging was rendered unfeasible during tracking with the OTS ${ }^{\text {vicra }}$ while fluorescence imaging became possible during tracking with the synchronized OTS ${ }^{\text {orthos }}$.

\subsection{Gated Tracking Pulse and Fluorescence Imaging for PDE}

In an attempt to completely remove the interference during fluorescence imaging, including the background illumination, an alternating gated tracking and fluorescence imaging setup was pursued. Unfortunately, it was not possible to identify a "dead-imaging-moment" capable of harboring the OTS ${ }^{\text {orthos }}$ tracking pulse. Consequently, we were not able to get rid of the stable background illumination.

\section{Discussion}

In this study we have investigated possible solutions to solve the interference issues found during the navigation of clinical
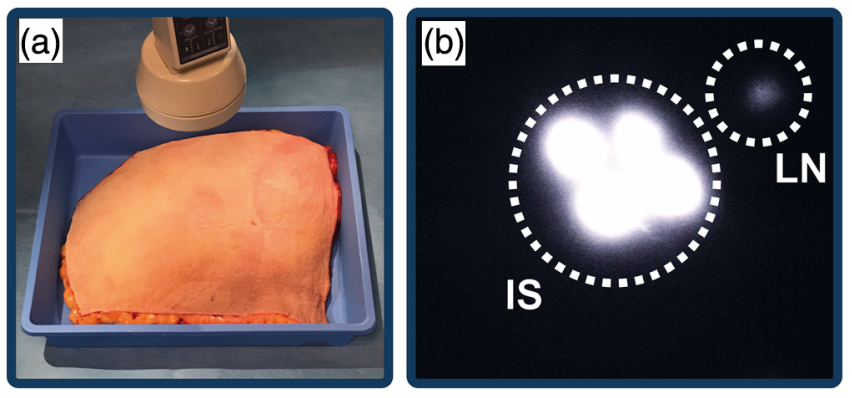

No tracking:

mean MPI = 28.6 counts; SDIV $=0.7$ counts $/ \mathrm{s}$

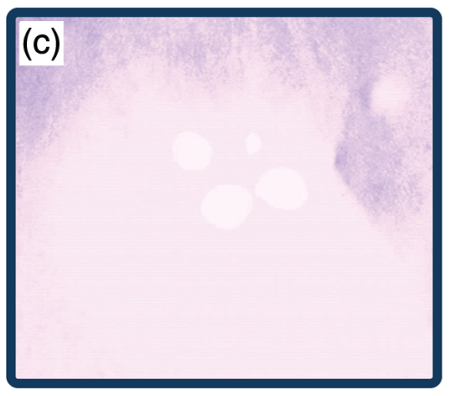

OTS vicra:

mean MPI = 205.4 counts; SDIV $=3501.6$ counts $/ \mathrm{s}$

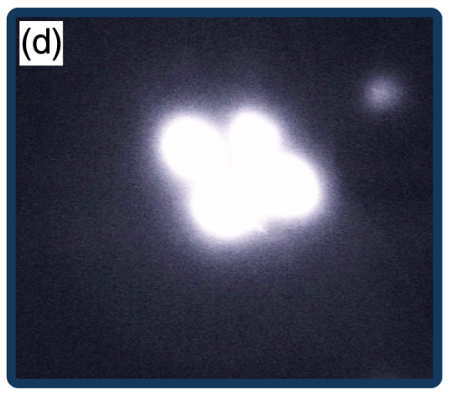

OTSorthos:

mean MPI = 44.0 counts; SDIV $=1.5$ counts $/ \mathrm{s}$

Fig. 8 Evaluation of optical tracking during fluorescence imaging for human tissue. (a) Excised human tissue with the PDE camera situated $15 \mathrm{~cm}$ above the tissue. (b) Fluorescence imaging without any tracking. Both injection site (IS) and sentinel lymph node (LN) are visible. (c, d) Example video frames for fluorescence imaging during tracking with the (c) OTS vicra and (d) synchronized OTS ${ }^{\text {orthos. }}$ 
fluorescence cameras using NIR optical tracking in open surgery. The interference was caused by a number of features: (1) the spectral overlap between the ICG emission light and the NIR OTS light, (2) the OTS light intensity, (3) the OTS duty cycle, (4) the OTS frequency, (5) the fluorescence camera recording frequency, and (6) the camera sensitivity. As we have also observed during our previous studies, ${ }^{4,5,8}$ this combination of features means that the severity of interference varied between the type of fluorescence camera used. More specifically, the observed interference with the OTS ${ }^{\text {vicra }}$ completely obstructed fluorescence imaging with the PDE camera while its influence on the imaging output of the VITOM camera was only minor.

Matching the tracking light pulse frequency with the specific camera imaging frequency, or a multiplication thereof, supported a drastic decrease in the dynamic character of the OTS interference. This approach did not solve the stable background signal. Further reduction of this background signal could be realized by minimizing the OTS pulse intensity and duty cycle. It appears that gating of the OTS tracking pulse and fluorescence image capture will provide outcome. For this to work, a fluorescence camera with a (controllable) long enough "dead-imaging-moment" is needed. As shown in Fig. 4 and Table 1, there is little room to decrease the spectral overlap by separating the current OTS spectrum from the emission of dyes that fluoresce in the NIR spectrum. This said, successful use of non-NIR dyes for surgical guidance has been reported, e.g., for fluorescein (emission maximum at $530 \mathrm{~nm}$ ), 5-aminolevulinate/protoporphyrin IX (emission maximum at $635 \mathrm{~nm}$ ), and Cy5 (emission maximum at $670 \mathrm{~nm}$ ). ${ }^{12,16-18}$ When such dyes are used in combination with clinically available far-red fluorescence camera systems, ${ }^{12,19}$ no overlap with the OTS will occur, allowing navigated camera positioning during open surgery.

Instead of changing the fluorescent dye used to create surgical guidance, one could also opt for using an alternative OTS with a light spectrum that does not overlap with the surgical fluorescence cameras. Unfortunately, most (clinical grade) systems use the NIR spectrum to prevent disturbance of the visible operating room lighting. ${ }^{20}$ Research grade OTS systems are already available that employ, e.g., CWL $940 \mathrm{~nm}$ NIR wavelengths ${ }^{20}$ which is higher than the OTS spectra used in this study. Next to NIR optical tracking, other tracking techniques might also provide outcome for the navigation of fluorescence cameras (e.g., electromagnetic tracking, mechanical tracking, and vision-based tracking). ${ }^{6,9,21,22}$ Of these methods, visionbased tracking seems to be optimally suited for fluorescence cameras when combined with (fluorescent) skin fiducials, detectable with the camera. ${ }^{23}$ Most realistically a combination of tracking technologies will provide outcome. ${ }^{24,25}$

Next to the fluorescence cameras that are currently available in the clinic, this research also supports the navigation of future surgical fluorescence imaging devices; e.g., fluorescence goggles and head-mounted displays. ${ }^{26}$

\section{Conclusion}

We have characterized the different aspects underlying the OTS interference during the navigation of NIR fluorescence cameras and have identified engineering routes that help solve these issues. With that a basis has been created to engineer OTSs specifically tailored toward the navigation of fluorescence cameras during open surgery.
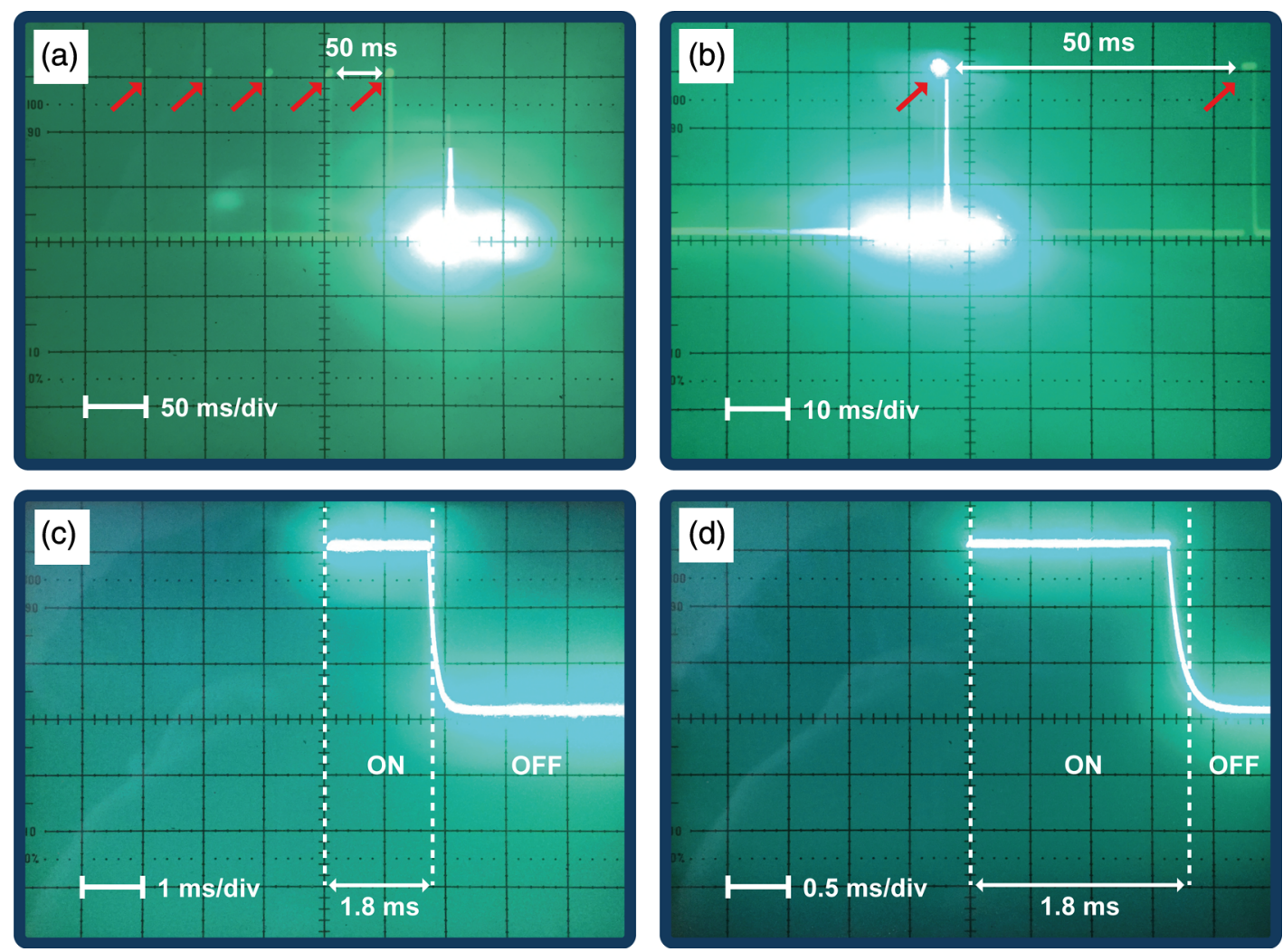

Fig. 9 Temporal characterization OTS vicra light pulse. Oscilloscope screens are shown at different magnifications. 


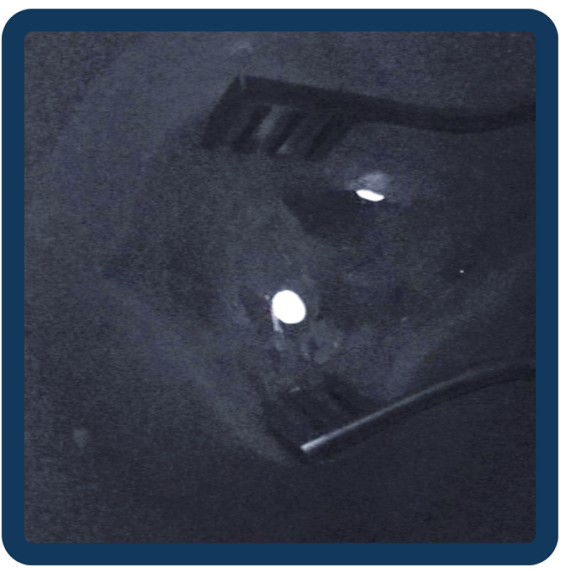

Fig. 10 Video example of OTS ${ }^{\text {vicra }}$-induced interference in the surgical fluorescence phantom navigation of PDE fluorescence camera (Video 3, MPEG, 5.8 MB) [URL: https://doi.org/10.1117/1.JBO.23.5 .056003.3])

\section{Appendix A: Spectral and Temporal Characterization}

A Horiba Jobin Yvon VS140 linear array fiber spectrometer (Horiba Ltd., Kyoto, Japan) with a 1-m optical fiber (M15L01, Thorlabs Inc., Newton, New Jersey) was used to spectrally characterize the excitation light of the different fluorescence cameras and the OTSs used.

To determine both the OTS pulse frequency and its duty cycle, a light-to-voltage setup was used. This setup consisted of an optical light-to-voltage sensor (TSL 250R, TAOS Inc., Plano, Texas), an analog $50 \mathrm{MHz}$ oscilloscope (no. 2225, Tektronix Inc., Beaverton, Oregon), and a direct current power supply (PS613 HQ POWER, Velleman NV, Gavere, Belgium). An example for the OTS ${ }^{\text {vicra }}$ is shown in Fig. 9.

\section{Appendix B: Surgical Fluorescence Phantom Details}

The fluorescent beads incorporated in the surgical fluorescence phantoms were created by mixing an ICG solution with an

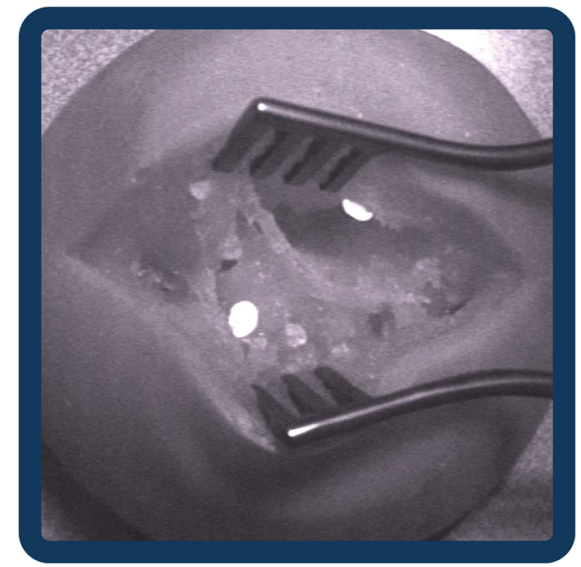

Fig. 12 Video example of OTS ${ }^{\text {orthos }}$-induced interference in the surgical fluorescence phantom navigation of PDE fluorescence camera (Video 4, MPEG, 13.4 MB [URL: https://doi.org/10.1117/1.JBO.23.5 .056003.4]).

epoxy resin (epoxy resin $\mathrm{C}, \mathrm{R} \& \mathrm{G}$ Faserverbundwerkstoffe $\mathrm{GmbH}$, Waldenbuch, Germany) using the following ratio: 10:6:1 for epoxy resin, epoxy hardener, and ICG solution, respectively. The ICG solution comprised of $1 \mathrm{mg} / \mathrm{mL}$ ICG dissolved in methanol. The effective ICG concentration in the beads was therefore $60 \mu \mathrm{g} / \mathrm{mL}$, which roughly presents a clinically relevant and detectable ICG concentration. ${ }^{8}$

\section{Appendix C: Visualization of OTS-Induced Interference during the In Vivo and Phantom Application}

An example of the OTS-induced interference during in vivo application of the navigated PDE fluorescence camera is shown in Figs. 1 and 2. This is based on data published by KleinJan et al., where the navigated PDE camera was demonstrated during sentinel lymph node biopsy in five penile cancer patients. ${ }^{4}$ Interference observed in the surgical fluorescence phantoms is illustrated for the OTS ${ }^{\text {vicra }}$ in Figs. 10 and 11, and for the

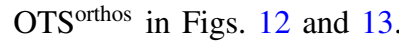

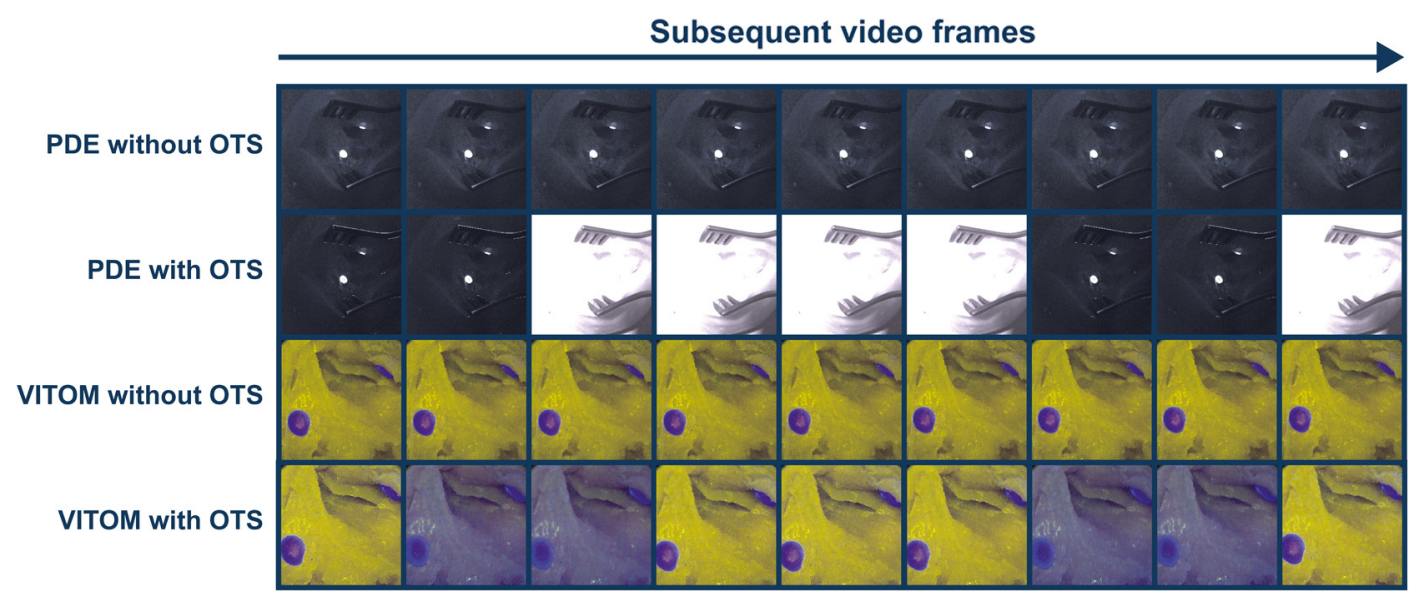

Fig. 11 Video images of OTS vicra-induced interference during fluorescence imaging in the open surgical phantom. Top to bottom shows video image sequences of the surgical fluorescence phantom for the PDE without the OTS vicra, PDE with the OTS vicra, VITOM without the OTS vicra, and VITOM with the OTS ${ }^{\text {vicra }}$. The OTS vicra was positioned at a distance of $130 \mathrm{~cm}$. 


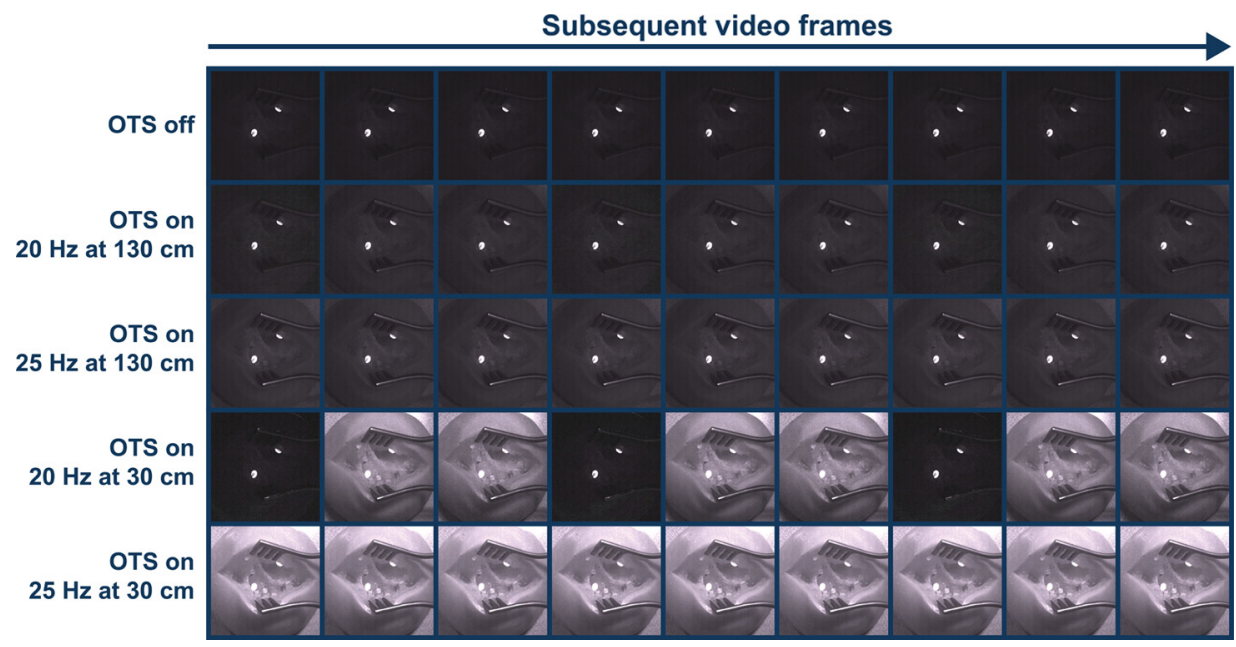

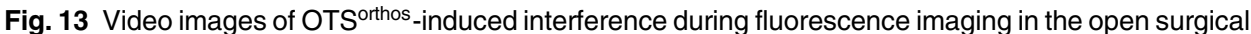
phantom. Top to bottom shows video image sequences of the surgical fluorescence phantom for the PDE without the OTS ${ }^{\text {orthos }}$ and with the OTS ${ }^{\text {orthos }}$ at different settings and distances.
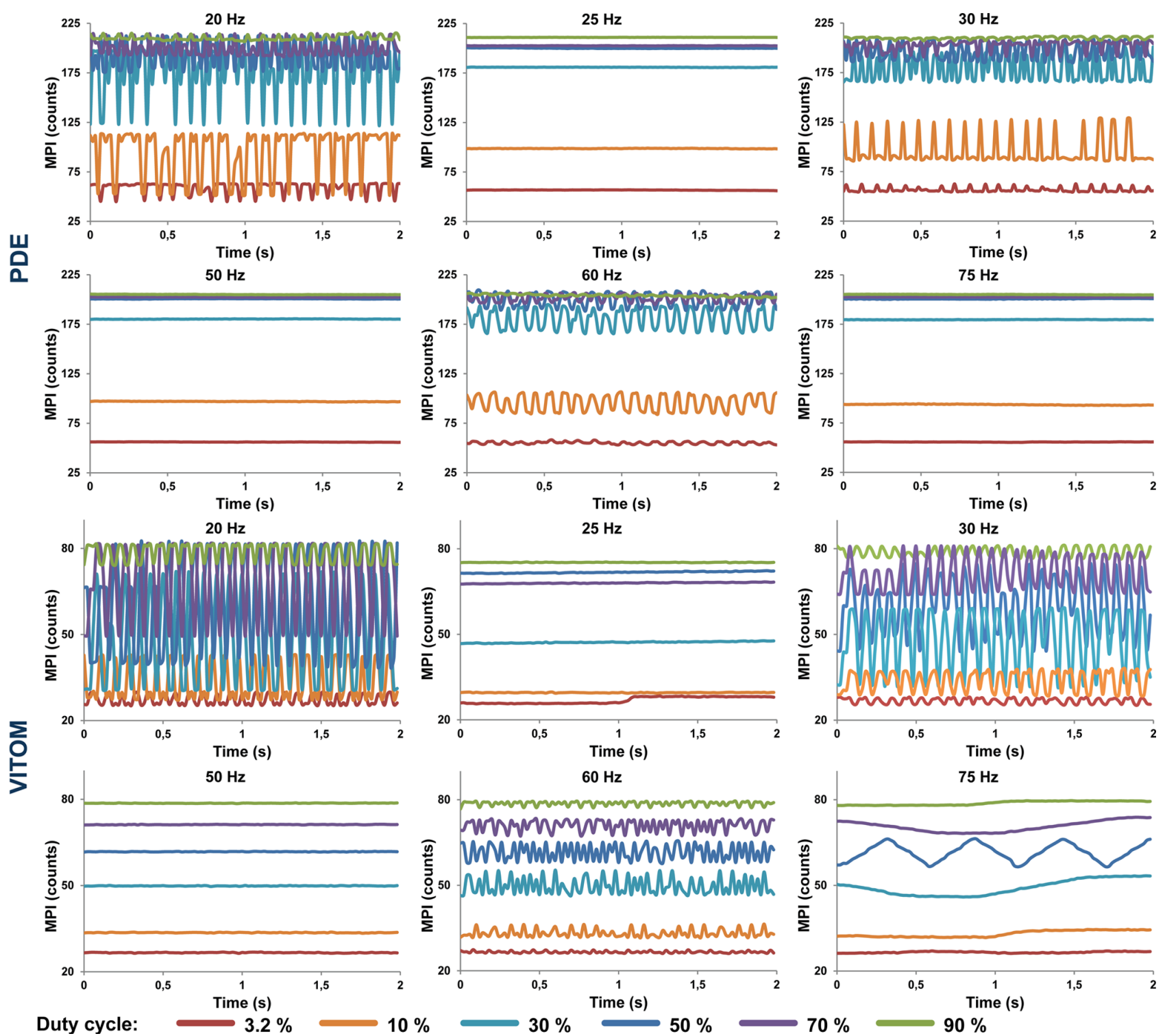

Fig. 14 Mean pixel intensity for interference induced by the LED setup at various pulse frequency and duty cycle settings using both the PDE and VITOM. 


\section{Appendix D: Detailed Overview of the MPI Measurements for a Modular Tracking Frequency and Duty Cycle}

A detailed overview of the MPI measurements obtained at different tracking frequency and duty cycle settings for the LED setup are shown for both the PDE and VITOM camera in Fig. 14.

\section{Disclosures}

The authors have no relevant financial interests in this manuscript and no potential conflicts of interest to disclose.

\section{Acknowledgments}

This work was supported by a European Research Council grant (ERC-advanced grant with grant no. 323105) and an NWOSTW-VIDI grant (grant no. STW BGT11272). Material support was provided by SurgicEye (SurgicEye, Munich, Germany), allowing the authors to use the OTS ${ }^{\text {orthos }}$ in this study, and by Hamamatsu (Hamamatsu Photonics K.K., Hamamatsu, Japan), lending the authors a PDE fluorescence camera for this study.

\section{References}

1. F. W. B. van Leeuwen, J. C. Hardwick, and A. R. van Erkel, "Luminescence-based imaging approaches in the field of interventional molecular imaging," Radiology 276(1), 12-29 (2015).

2. I. Stoffels et al., "Intraoperative fluorescence imaging for sentinel lymph node detection: prospective clinical trial to compare the usefulness of indocyanine green vs technetium Tc $99 \mathrm{~m}$ for identification of sentinel lymph nodes," JAMA Surg. 150(7), 617-623 (2015).

3. O. R. Brouwer et al., "Comparing the hybrid fluorescent-radioactive tracer indocyanine green-99mTc-nanocolloid with 99mTc-nanocolloid for sentinel node identification: a validation study using lymphoscintigraphy and SPECT/CT,' J. Nucl. Med. 53(7), 1034-1040 (2012).

4. G. H. KleinJan et al., "Toward (hybrid) navigation of a fluorescence camera in an open surgery setting," J. Nucl. Med. 57(10), 1650-1653 (2016).

5. M. N. van Oosterom et al., "Navigation of fluorescence cameras during soft-tissue surgery-is it possible to use a single navigation setup for various open and laparoscopic urological surgery applications?" J. Urol. 199, 1061-1068 (2018).

6. M. N. van Oosterom et al., "Computer-assisted surgery: virtual-and augmented-reality displays for navigation during urological interventions," Curr. Opin. Urol. 28(2), 205-213 (2018).

7. O. R. Brouwer et al., "Image navigation as a means to expand the boundaries of fluorescence-guided surgery," Phys. Med. Biol. 57(10), 3123-3136 (2012).

8. M. N. van Oosterom et al., "Navigation of a robot-integrated fluorescence laparoscope in preoperative SPECT/CT and intraoperative freehand SPECT imaging data: a phantom study," J. Biomed. Opt. 21(8), 086008 (2016).

9. P. Waelkens et al., "Surgical navigation: an overview of the state-of-theart clinical applications," in Radioguided Surgery, K. Herrmann, O. E. Nieweg, and S. P. Povoski, Eds., pp. 57-73, Springer International Publishing, Switzerland (2016).
10. B. Zhu and E. Sevick-Muraca, "A review of performance of near-infrared fluorescence imaging devices used in clinical studies," Br. J. Radiol. 88(1045), 20140547 (2015).

11. G. H. KleinJan et al., "Optimisation of fluorescence guidance during robot-assisted laparoscopic sentinel node biopsy for prostate cancer," Eur. Urol. 66(6), 991-998 (2014).

12. D. M. van Willigen et al., "Multispectral fluorescence guided surgery; a feasibility study in a phantom using a clinical-grade laparoscopic camera system," Am. J. Nucl. Med. Mol. Imaging 7(3), 138-147 (2017).

13. G. H. KleinJan et al., "Fluorescence guided surgery and tracer-dose, fact or fiction?" Eur. J. Nucl. Med. Mol. Imaging 43(10), 1857-1867 (2016).

14. A. V. DSouza et al., "Review of fluorescence guided surgery systems: identification of key performance capabilities beyond indocyanine green imaging," J. Biomed. Opt. 21(8), 080901 (2016).

15. S. van der Wal et al., "Synthesis and systematic evaluation of symmetric sulfonated centrally C-C bonded cyanine near-infrared dyes for protein labelling," Dyes Pigm. 132, 7-19 (2016).

16. F. Acerbi et al., "Fluorescein-guided surgery for malignant gliomas: a review," Neurosurg. Rev. 37(4), 547-557 (2014).

17. S. E. Craig et al., "Fluorescent-guided surgical resection of glioma with targeted molecular imaging agents: a literature review," World Neurosurg. 90, 154-163 (2016).

18. N. S. van den Berg, F. W. B. van Leeuwen, and H. G. van der Poel, "Fluorescence guidance in urologic surgery," Curr. Opin. Urol. 22(2), 109-120 (2012).

19. J. Burggraaf et al., "Detection of colorectal polyps in humans using an intravenously administered fluorescent peptide targeted against c-Met," Nat. Med. 21(8), 955-961 (2015).

20. R. Yang et al., "Design of an accurate near infrared optical tracking system in surgical navigation," J. Lightwave Technol. 31(2), 223-231 (2013).

21. S. Bernhardt et al., "The status of augmented reality in laparoscopic surgery as of 2016," Med. Image Anal. 37, 66-90 (2017).

22. W. Birkfellner et al., "Tracking devices," in Image-Guided Interventions, T. Peters and K. Cleary, Eds., pp. 23-44, Springer, Boston, Massachusetts (2008).

23. E. Wild et al., "Robust augmented reality guidance with fluorescent markers in laparoscopic surgery," Int. J. Comput. Assisted Radiol. Surg. 11(6), 899-907 (2016).

24. B. Fuerst et al., "First robotic SPECT for minimally invasive sentinel lymph node mapping," IEEE Trans. Med. Imaging 35(3), 830-838 (2016).

25. A. Hughes-Hallett et al., "Augmented reality partial nephrectomy: examining the current status and future perspectives," Urology $\mathbf{8 3}(2)$, 266-273 (2014).

26. S. B. Mondal et al., "Optical see-through cancer vision goggles enable direct patient visualization and real-time fluorescence-guided oncologic surgery," Ann. Surg. Oncol. 24, 1897-1903 (2017).

Matthias N. van Oosterom received his MSc degree in applied physics from the Delft University of Technology in 2014. Currently, he is a PhD student at the Departments of Radiology and Surgery of the Leiden University Medical Center. Since his studies, he has been active (often in close collaboration with industry) in the development of molecular imaging modalities, for example by combining nuclear and optical imaging in hybrid devices and exploring new surgical navigation concepts.

Biographies for the authors are not available. 